\title{
Verknüpfung von Identifikationstechnologien an Flurförderzeugen mit übergeordneten Lagerverwaltungssystemen mittels standardisierter IT- Integration
}

\author{
DIPL.-INFORM.WIRT KAI BECKHAUS, \\ PROF. DR.-ING. WILLIBALD A. GÜNTHNER \\ LEHRSTUHL FÜR FÖRDERTECHNIK MATERIALFLUSS LOGISTIK, TU MÜNCHEN
}

\section{Zusammenfassung}

Prozessverbesserungsvorschläge durch den Einsatz von RFID und anderen neuen Technologien können die Effizienz in der Intralogistik steigern indem manuelle Kontrollschritte entfallen. Zusätzlich wird eine Verringerung der Fehlerquote erreicht und dadurch z.B. Fehlverladungen vermieden. Die Prozesssteuerung der Staplerfahrer und die Datenverarbeitung führen meist Lagerverwaltungssysteme durch. Bisher erfüllt dabei projektindividuelle Software die Vermittlerrolle zwischen der RFID-Hardware auf den Flurförderzeugen und der Dateneingabe. Eine systematische und umfassende Integration in die prozessführenden Lagerverwaltungssysteme auf dieser dezentralen Ebene wurde in der Vergangenheit vernachlässigt. Diese ist allerdings unabdingbar, um das gesamte Potenzial der Prozessverbesserung auszuschöpfen. Der zugrunde liegende Beitrag betrachtet eine standardisierte softwaretechnische Integration und leitet ein Konzept her, welches anhand eines Umsetzungsbeispiels validiert wurde.

\begin{abstract}
Process improvements by using RFID and other new technologies can help to gain efficiency in the area of intralogistics. The main levers thereby are the abolition of manual control scans and the reduced error-ratio - for example also leading to less deficit-loadings. The process guidance of truck drivers and the data handling is regularly done by WarehouseManagement-Systems. So far, project individual software has thereby taking the mediator-role between RFID-hardware on the trucks and the data entry point. A systematic and holistic integration into the Warehouse-Management-Systems has not yet been applied on this decentral view - even though, it is essential for utilizing the complete potential of process improvements. This article discusses a standardised software integration and suggests an integration concept, which will be validated by an application example.
\end{abstract}




\section{Stand der Technik}

Kaum ein größeres Lager arbeitet heutzutage ohne Lagerverwaltungssystem (LVS). Dabei lassen sich Betriebe unterscheiden, die noch "offline" arbeiten und vom LVS-Server papierbasierte Listen drucken, die der Fahrer nach Abarbeitung zurück-quittiert sowie "online" Systeme, die jeden Auftrag über ein Funknetz an ein Datenfunkterminal mit LVS-Client schicken. Die Staplerfahrer können dort die Aufträge lesen, ausführen und in Echtzeit zurückquittieren. Im Folgenden ist die weitaus häufiger anzutreffende "online" Version Gegenstand der Diskussion.

Um sich bei der Rückmeldung einer Aktion nicht alleine auf die Bestätigung des Staplerfahrers zu verlassen, werden in den meisten Fällen Identifikations- und Kontrollschritte mittels Barcodescanner vollzogen. Dadurch ergeben sich im wesentlichen drei Vorteile:

- $\quad$ Vereinfachung der Informationseingabe (Komfort und Zeit)

- $\quad$ Kontrolle des Fahrers

- Verringerung der Verwechslungsgefahr

Um die Prozesse des Lagerbetriebs bestmöglich zu unterstützen, sind in der Regel mehrere Kontrollvorgänge nötig. Abbildung 1 zeigt einen vereinfachten typischen Ablauf für Ein- und Auslagerungen. Bei jeder Aktion sind zur Verbuchung der Palettenbewegung die Identifikation der Palette und des Abgabeorts zentral.

Um den Identifikationsprozess weiter zu verbessern können Radio-Frequenz-Identifikationssysteme (RFID) Teilschritte übernehmen. Der Einsatz einer RFID-Lösung hat den Vorteil, dass eine Verwechslung durch Scannen des falschen Barcodes ausgeschlossen ist und der Fahrer den Identifikationsprozess nicht manuell durchführen muss. Vielmehr werden die Daten automatisch erhoben. An Flurförderzeugen werden RFID-Schreib-/Lesegeräte dazu entweder am Gabelrücken angebracht oder in die Gabelzinken integriert (vgl. [Jun-09], [Gün-09]). Es können sowohl Paletten als auch Lagerfächer erkannt werden. Eine Voraussetzung dazu ist die Ergänzung der entsprechenden Barcodes um RFID-Transponder. 
Einlagerung

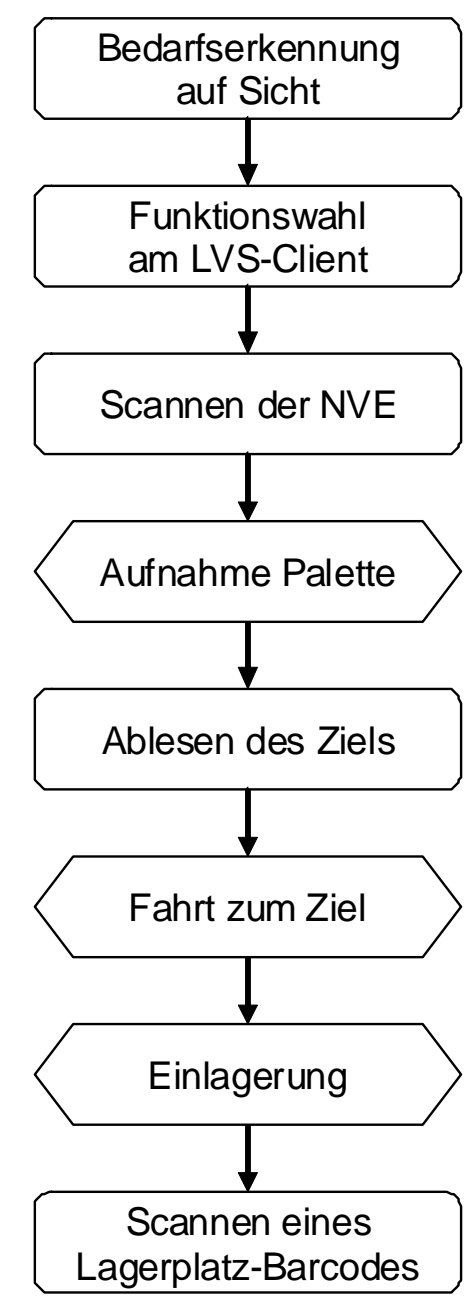

Auslagerung

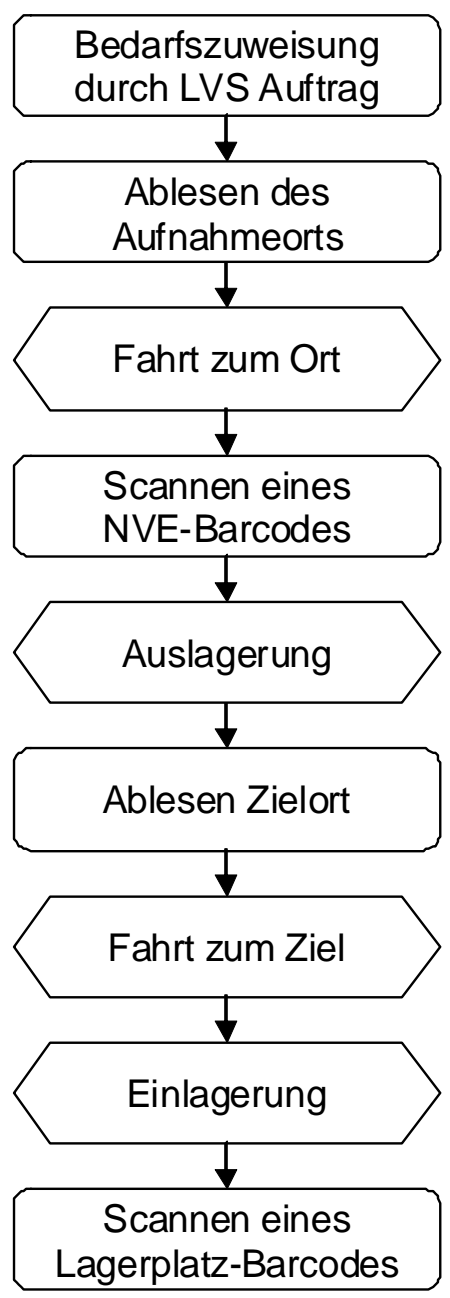

Datenaktion /

Informationsaktion

\section{Abbildung 1: Vereinfachte Standardprozesse des Lagerbetriebs}

Der Informationsfluss beim Identifikationsprozess läuft vom Datenträger (Barcode, Transponder) zum Lagerverwaltungssystem. Der Einsatz einer RFID-Hardware kann dabei einen Unterschied in der Informationsintegration bewirken. Ein Barcodescanner fungiert entweder direkt via USB oder indirekt via serieller Schnittstelle und Treiber wie eine extern angeschlossene Tastatur. Das Lagerverwaltungssystem kann also an sich nicht unterscheiden, ob es die Daten durch die Eingabe des Fahrers (sofern ihm eine externe Tastatur oder Bildschirmtastatur bereitgestellt wird) oder dem Barcodescanner bekommt. Ein RFID-System hat den großen Unterschied, dass das Leseereignis in der Regel nicht durch den Fahrer, sondern automatisch ausgelöst wird. Bislang wurde eine dedizierte Betrachtung der IT-Integration von Wissenschaft und Praxis 
vernachlässigt. In den meisten Lösungen fungiert der RFID-Leser wie ein Barcodescanner und gibt die Leseergebnisse auch per Tastatureinschleifung an das Lagerverwaltungssystem weiter. Somit ist es den Herstellern von RFID-Lösungen möglich ihre Lösung als "zu allen Lagerverwaltungssystemen kompatibel" zu vermarkten. Bei genauer Betrachtung fällt allerdings schnell auf, dass dies zu Problemen führen kann. Da die Integration nur unidirektional stattfindet, kann das RFID-Gesamtsystem nicht wissen, welche Eingabe das Lagerverwaltungssystem als nächstes benötigt. Folgendes Ablaufbeispiel zeigt einen möglichen Fehlerverlauf:

1. Das LVS fordert eine Bestätigung einer Paletten-NVE

2. Der Fahrer kommt dem RFID-System zuvor und scannt die Palette bereits mit dem Barcodescanner, welcher als Backup-Lösung noch installiert ist

3. Das LVS verbucht den Auftrag und zeigt dem Fahrer eine beliebige neue Maske an.

4. Das RFID-System liest nun die Palette und gibt die gelesene NVE Nummer per Tastenschlag an die LVS Maske weiter.

5. Die Tastenschläge kommen beim LVS an, führen aber zu einer willkürlichen und falschen Eingabe.

Ein weiterer Problempunkt bei der bisherigen Integration von RFID-Systemen ist die Ausgestaltung der entsprechenden Anbindungssoftware. $\mathrm{Zu}$ einer RFID-Hardware legt der Hersteller meistens einen Treiber in Form einer Funktionsbibliothek bei. Das Abgreifen der Daten und die Weiterverarbeitung bis zur Weiterleitung stellen in der Regel keine sehr umfangreiche Aufgabe dar. Aus diesem Grund werden oft projektindividuelle Softwarelösungen entwickelt, die schnell erstellt sind, aber häufig keinen Standards entsprechen. Eine Alternative wäre der Einsatz von sogenannten Middleware-Systemen, welche sowohl zur untergeordneten Ebene der RFID-Hardware als auch zur übergeordneten Ebene der Datenverwaltungssystemen Schnittstellen bereitstellen. Die am Markt verfügbaren Systeme wie z.B. der Tagpilot der Fa. Silverstroke GmbH [Sil-09] oder das Produkt You-R OPEN der Fa. RF-iT Solutions GmbH [RFi09] sind allerdings sehr umfangreich und bieten weit mehr Funktionalität als eigentlich benötigt. Dementsprechend schwierig ist der Einsatz auf den dezentralen Datenfunkterminals, welche meist über wenig Rechenleistung verfügen.

\section{Problemstellung}

Um die aufgezeigte Lücke der mangelhaften Ausgestaltung der Anbindungssoftware zu füllen bedarf es einer Lösung, welche sowohl Middleware-Funktionalitäten (Schnittstellen, Datenfilterung und Umwandlungen) in den wesentlichen Punkten übernimmt als auch die logistische Prozesssteuerung unterstützen kann. Hierzu ist es notwendig eine bidirektionale Kommunikation zwischen Lagerverwaltung und Middleware zu planen, damit das technische System zu jedem Zeitpunkt den Status des prozessführenden Lagerverwaltungssystems kennt. Nur so kann eine weitgehende Integration erfolgen und können Fehler vermieden werden. Ein weiteres Problem einer nur unidirektional geplanten Kommunikation ist, dass eine Übermittlung von "Soll-Daten" nicht möglich ist. Werden beispielsweise mit einer Gabelzinkenantenne mehrere Paletten aufgenommen, so kann die Information welche Paletten erwartet werden dazu genutzt werden dem Fahrer eine graphische Ansicht zu bieten. Durch diese Informationsübermittlung von Lagerverwaltung zur Middleware ergeben sich demnach neue Ansätze und Funktionalitäten für eine grundlegend geänderte Vernetzung der Systeme, die bisher nicht berücksichtigt werden. 


\section{Anforderungen an ein Stapler-Middleware-System}

Ein für den dezentralen Einsatz auf Flurförderzeugen geeignetes Middleware System muss drei Kern-Anforderungen erfüllen.

- $\quad$ Erstens muss es niedrige Systemvoraussetzungen haben, da es auf einem Datenfunk Computer ausgeführt werden soll.

- Z Zweitens muss es einem offenen, standardisierten und modularen Aufbau folgen, um universal einsetzbar und skalierbar zu sein.

- $\quad$ Drittens sollte es ein funktionales Grundgerüst mitbringen, welches eine geeignete Anzahl an Funktionen und Inhalten zur Verfügung stellt, um mit geringen Anteilen an individueller Programmierung einsetzbar zu sein.

Die Leistungsfähigkeit von Datenfunk-PCs läuft in der Regel mindestens zwei Jahre hinter den am Markt verfügbaren mobilen Laptops im Standardpreissegment hinterher. In allen vier wichtigen Bewertungsfaktoren Prozessor-Leistung, Arbeitsspeicher, Datenspeicher (Festplatte oder SD-Karte) und Betriebssystem ist mit Leistungsbeschränkungen zu rechnen. Der Grund für diese Entwicklung liegt in der Anforderungsverschiebung weg von den benannten Bewertungsfaktoren - da die bisher zum Einsatz kommenden LVS-Client-Applikationen mit wenig Anforderungen auskommen - hin zu Anforderungen in Bezug auf den Einsatz unter erschwerten physischen Bedingungen - z.B. durch die Forderung von IP-Schutzklassen, Vibrationsresistenz und EMV Verträglichkeit. Ein Middlewaresystem muss sich daher mit geringem Ressourcenbedarf begnügen und sollte auch geringe Anforderungen in Bezug auf vorhandene Komponenten wie z.B. Java Runtime Umgebung oder Microsoft .NET Framework besitzen.

Die Anforderungen an die prinzipielle Ausgestaltung einer Middleware Software leiten sich aus verschiedenen End-Anforderungen und Schwachstellen von bisherigen Systemen ab. Die häufig bisher für diesen Zweck zum Einsatz kommenden individuellen Softwarelösungen werfen verschiedene Probleme auf:

- Individuell gestalteter Quellcode, ggf. sogar Abhängigkeit von Software-Entwicklern

- $\quad$ Geringe Modularität

- $\quad$ Change-Request Umsetzungszeit hoch

- $\quad$ Schwer erweiterbar

- Eingeschränkte Wiederverwendungsmöglichkeit von Programmteilen für ähnliche Projekte

Unter Modularität eines Softwaresystems wird dabei die Ausgestaltung in gekapselten Teilen verstanden, welche klar von einander über eine Schnittstelle abgegrenzt sind (vgl. z.B. Definition Begriff "Modul" [Bro-86]).

Aus den genannten Schwachstellen lassen sich die drei in Abbildung 2 gezeigten primären Anforderungen an die Softwaregestaltung herleiten. 


\section{Anforderungsdreieck der}

Softwaregestaltung

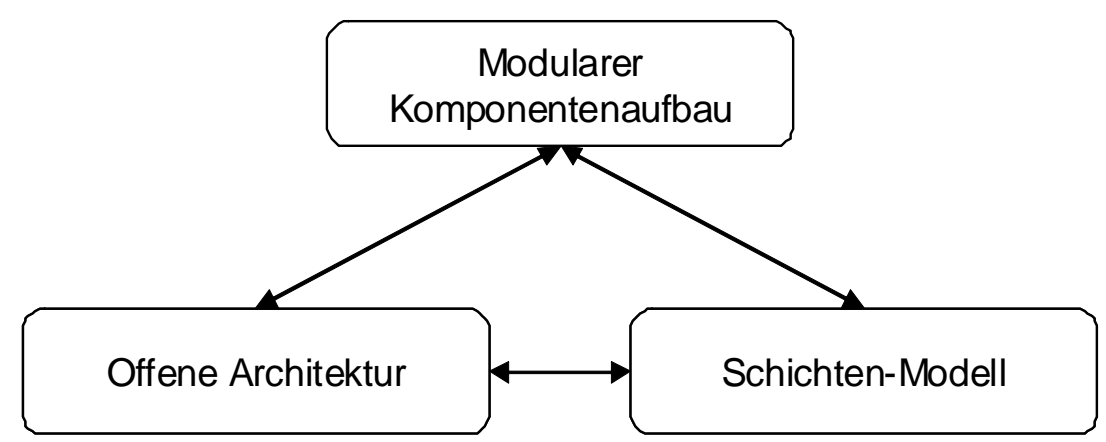

Abbildung 2: Anforderungsdreieck der Softwaregestaltung

Der modulare Komponentenaufbau ermöglicht das Kapseln funktional abgegrenzter Teilgebiete in einzelne Software Komponenten. Die einzelnen Komponenten müssen über eine geeignete Kommunikationsschnittstelle miteinander verbunden werden. Eng verbunden mit dieser Anforderung ist die "Offene Architektur", welche spezifiziert, dass es externen Personen möglich sein muss Komponenten für das System zu entwickeln. Gewisse Teile des Quellcodes oder zumindest eine sogenannte Application-Programming-Interface (API) müssen also frei verfügbar sein. Die technische Ausgestaltung des Gesamtsystems soll abschließend einem SchichtenModell folgen. Hierunter ist eine stufenweise Verarbeitung der Daten zu verstehen. So wird zum Beispiel ein von einem RFID-Reader gelesener Transponder zunächst von einem Treiber auf dem Datenfunkterminal zur Verfügung gestellt. Hier wird er von einer Anbindungskomponente der Middleware abgefragt, danach gefiltert und ggf. um Zusatzinformationen wie z.B. einem Zeitstempel oder der Lesehäufigkeit ergänzt. Folgt man dem Schichtenmodell weiter, so wird die Verarbeitung nun an eine Prozesskomponente übergeben, welche den richtigen Zeitpunkt zur Übergabe an das Lagerverwaltungssystem überwacht. Die technische Übergabe erfolgt dabei durch eine wiederum darüber liegende Schicht. 


\section{Schichtenmodell}

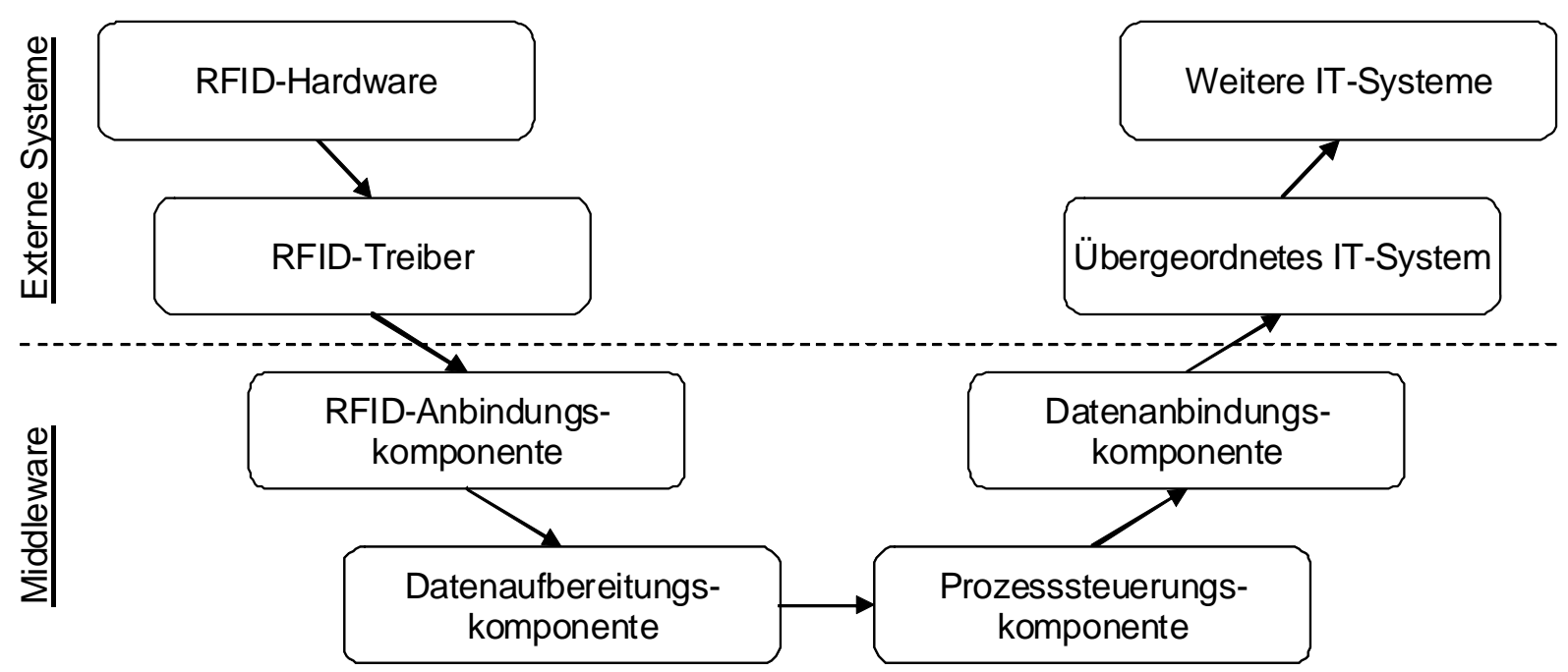

Abbildung 3: Schichtenmodell des Gesamtsystems

Durch die weite Verbreitung und hohe Bandbreiten des Internets ist es möglich Softwareanwendungen zu dezentralisieren und Service-orientierte Architekturen (SOA) zu nutzen. Eine allgemeingültige Definition des Begriffs, welcher erstmals in den neunziger Jahren genutzt wurde, gibt es nicht. Unter SOA kann man ein Software-Einsatz-Paradigma verstehen, welches zur Strukturierung dezentral verteilte Funktionen nutzt, die von verschiedenen Parteien (meist über das Internet) bereitgestellt werden (vgl. [Erl-06], [Las-09]). Die vorgeschlagene Anforderungsstruktur zur Gestaltung von Middlewaresystemen für Flurförderzeugen ähnelt in großen Teilen den SOA Konzepten. So lassen sich die Komponentenaufteilung und offene Gestaltung direkt übertragen.

Die nach geringen Systemvoraussetzungen und Modularität folgende dritte Grundanforderung beschreibt den Bedarf eines gewissen inhaltlichen Funktionsumfangs, der mindestens gegeben sein muss. Um die eingangs beschriebenen Nachteile der bisherigen unidirektionalen Anbindung auszugleichen ist es inhaltlich von zentraler Bedeutung eine Integration zu Lagerverwaltungssystemen in beide Richtungen vorzusehen. Dazu sind verschiedene Anbindungswege zu berücksichtigen und gekapselt abzubilden. Funktional besteht die wichtigste Aufgabe eines Middleware-Frameworks darin, dem nutzenden Programmierer wiederholende Routineaufgaben abzunehmen und eine geeignete Bibliothek an Standardfunktionen zur Benutzung mitzuliefern.

\section{Konzepterstellung und prototypische Umsetzung}

Zur Entwicklung eines Middleware Systems, welches den genannten Anforderungen gerecht wird, galt es zunächst die Wahl einer Programmierumgebung zu treffen. Eine Vergleichsanalyse ergab als geeignete Kompromisslösung die Verwendung des Microsoft .NET Frameworks in Version 1.1. Abbildung 4 zeigt die Vor- und Nachteile dieser Plattform. 


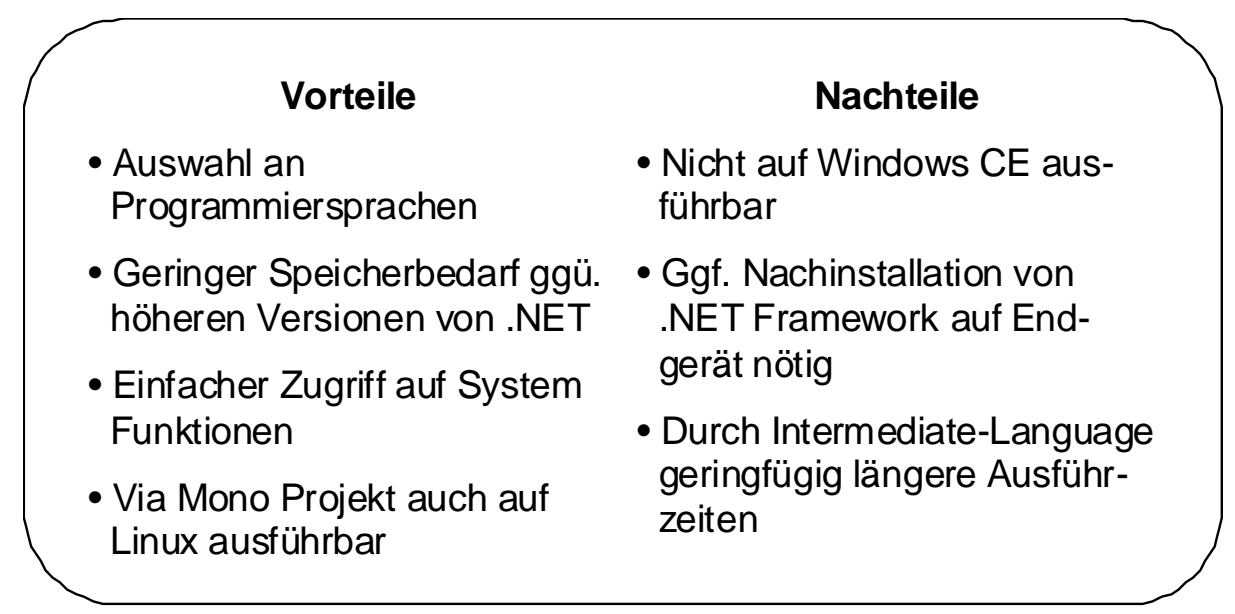

Abbildung 4: Abwägung Plattformwahl Microsoft .NET 1.1

Um den zentralen Anforderungen aus Abbildung 2 gerecht zu werden, wurde als Modularitätsebene eine Komponenten-Kapselung auf DLL Basis gewählt. Ein Hauptprogramm sucht sich dazu beim Start der Anwendung dynamisch die vorhandenen DLL-Komponenten und bindet diese daraufhin dynamisch ein. Das Hauptprogramm übernimmt nach der Initialisierungsphase nur noch die Funktion einer Nachrichtenstelle. Die gesamte Funktionalität wird von den Komponenten erbracht, welche untereinander mittels eines standardisierten Nachrichtenaustauschs kommunizieren und somit die Funktionalität abstimmen können. Dabei gibt es drei Nachrichtenarten: Anfrage, Antwort und Information. Die Kombination von Anfrage und Antwort Nachricht entspricht der Abbildung der SOA-Prinzipien. Benötigt eine Komponente eine Aktion einer anderen Komponente, so sendet sie die entsprechende Anfrage-Nachricht und erhält das Ergebnis als Antwort-Nachricht zurück. Der dritte Nachrichtentyp Information wird genutzt um einer Komponente Daten zu übertragen, die nicht direkt aus einer Anfrage resultieren. Inhaltliche Funktionalität stellen erste entwickelte Komponenten zur Verfügung. So wurden verschiedene technische Anbindungswege an Lagerverwaltungssysteme zunächst detailliert analysiert, dann entsprechende Anbindungswege spezifiziert und letztendlich umgesetzt. Das Ergebnis dieser Analyse ist ein Komponentensatz, welcher es Lagerverwaltungssystemen ermöglicht, via einer der folgenden Anbindungswege Daten an das Stapler-Middleware System zu übertragen:

$\begin{array}{ll}\text { - } & \text { Dateitransfer } \\ \text { - } & \text { Tcp/Ip Socket Verbindung } \\ \text { - } & \text { DLL-Datei oder EXE-Datei Aufruf } \\ & \text { Telnet }\end{array}$

Neben der technischen Ausgestaltung der Übertragung von LVS zur Middleware ist eine inhaltliche Spezifikation notwendig, um die Daten zwischen Sender und Empfänger auszutauschen. Hier kann ein für die verschiedenen logistischen Funktionen verwendeter Funktionskatalog auf XML Basis verwendet werden. Die Übertragung in der Rück-Richtung von Middleware zum Lagerverwaltungssystem kann entweder auf gleichem Weg in einem parallelen Kanal erfolgen oder wie in bisherigen Lösungen via Tastatur-Einschleifung vollzogen werden.

Auf der anderen Seite des Schichtenmodells wurden prototypisch RFID-Reader angebunden und eine Schnittstelle zur Fahrzeugsteuerung programmiert. Abbildung 5 zeigt die Architektur der

(C) 2010 Logistics Journal : Nicht referierte Veröffentlichungen - ISSN 1860-5923 Seite 8 
Umsetzung. Die einzelnen Komponenten der Middleware gliedern sich wie in einem Puzzle an das Hauptprogramm an und können durch einzelne Funktionalität in Kombination mit einem universalen Nachrichtenaustausch die Gesamtfunktionalität erbringen.

\section{Konzept-Architektur}

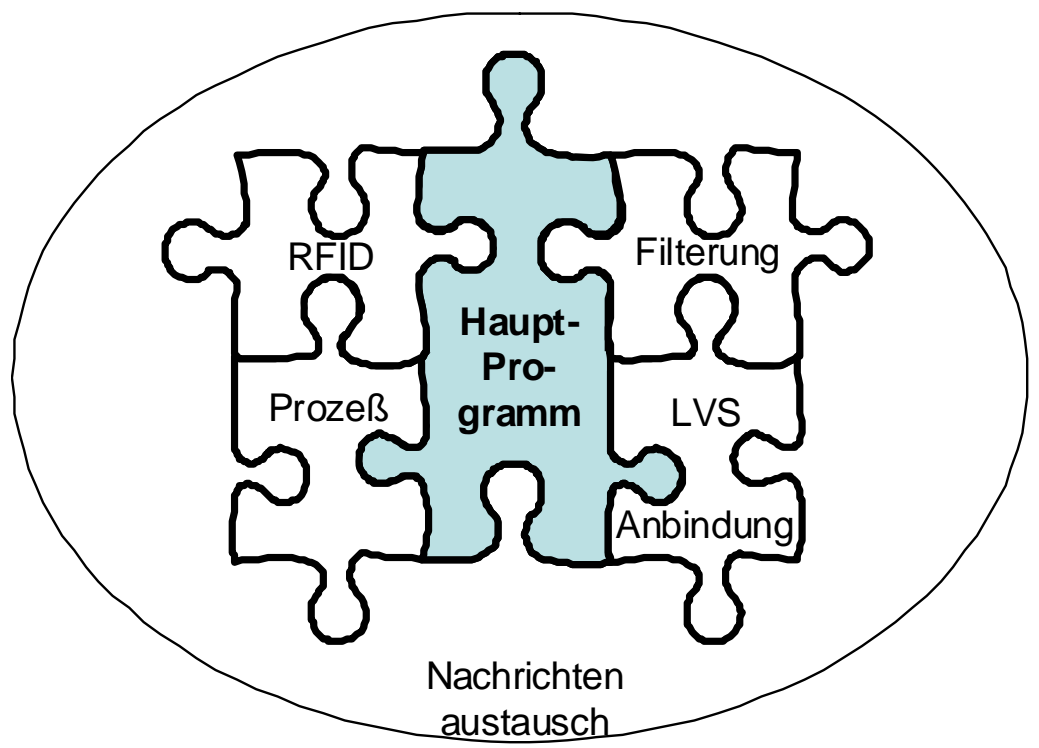

Abbildung 5: Architektur der Konzeptumsetzung als Puzzle-Darstellung

\section{Anwendungsbeispiel}

In einem Anwendungsprojekt bestand die Zielsetzung darin, ein Lagerverwaltungssystem mit der Fahrzeugsteuerung eines Schmalgang-Staplers zu verbinden. Der Stapler vom Typ EKX515 der Fa. Jungheinrich verfügt dabei über die Option "Lagernavigation", welche es ermöglicht halbautomatisch zum Zielplatz zu fahren und die gewünschte Ein- oder Auslagerung vorzunehmen [Rie-09]. Müsste der Fahrer den Zielplatz allerdings am Datenfunkterminal ablesen und dann "per Hand" dem Fahrzeug über ein Display mitteilen, wäre der zeitliche Vorteil der Lagernavigation kompensiert.

Durch die Stapler-Middleware, welche auf dem Datenfunkterminal im Hintergrund ausgeführt wird, wird diese Hürde überwunden. Eine Komponente der Middleware empfängt die Daten vom Lagerverwaltungsserver und übergibt sie einer Komponente, welche die Daten wiederum so aufbereitet, dass das Fahrzeug sie versteht. Sobald dieser Datensatz nun an die Komponente übermittelt wird, welche die Sprache der Fahrzeugsteuerung via seriellem Kabel zwischen Datenfunkterminal und Stapler spricht, ist der Informationsfluss vollzogen.

Zur technischen Realisierung der Anbindung an das Lagerverwaltungssystem standen verschiedene Wege zur Auswahl. Für die IT-Abteilung des Anwenders war es am wenigsten Aufwand ein separates Telegramm vom Server an das Terminal zu senden. Somit kam hier eine Tcp/Ip Socket Verbindungskomponente zum Einsatz. Abbildung 6 zeigt die neu geschaffene Systemlandschaft in dieser Projektinstallation. 


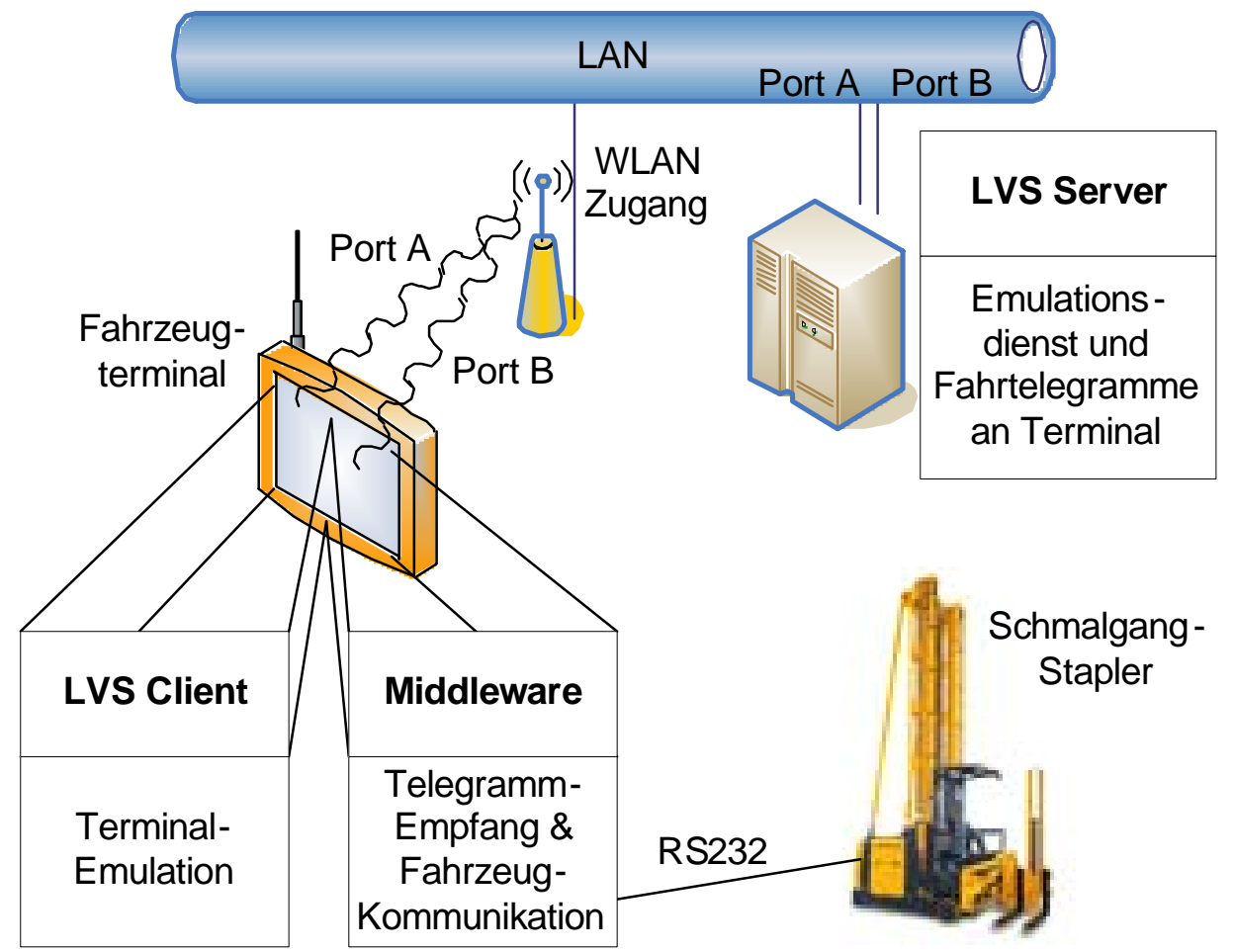

Abbildung 6: Systemlandschaft Anwendungsinstallation (Bildquelle Stapler: Jungheinrich)

Erste Messungen der Effizienzsteigerungen des Gesamtsystems zeigen, dass die Integration der Funktionen mit dem Lagerverwaltungssystem eine zweistellige Prozentzahl an Effizienzsteigerung im Palettenumschlag bewirkt [Rie-10]. Wie in Abbildung 7 veranschaulicht, amortisiert sich die einmalige Investition in der Regel bereits nach ein bis zwei Jahren, da die Zeiteinsparung und Qualitätssteigerung bei jeder Palettenbewegung ein hohes Nutzenpotenzial aufweisen.

\section{Wirtschaftlichkeitsbetrachtung}

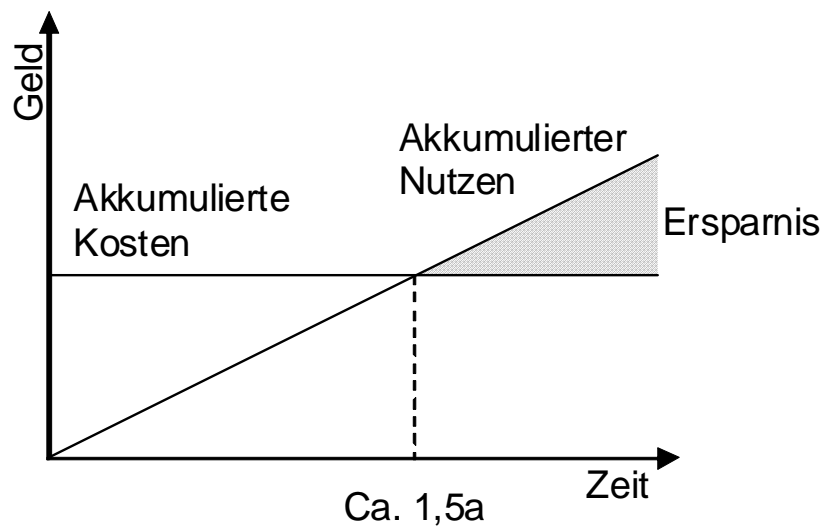

(C) 2010 Logistics Journal : Nicht referierte Veröffentlichungen - ISSN 1860-5923 


\section{Abbildung 7: Kosten / Nutzen Betrachtung}

\section{Zusammenfassung und Ausblick}

Um Identifikationstechnologien wie RFID sinnvoll einsetzen zu können, muss die technische Lösung sinnvoll in das prozessführende Lagerverwaltungssystem eingebunden werden. Eine unidirektionale Übermittlung der Leseergebnisse reicht dazu nicht aus. Integrationsprojekte sollten dazu standardisierte Softwarekomponenten individueller Programmierung vorziehen. So kann gewährleistet werden, dass Änderungen einfacher eingearbeitet werden können und auch die Softwareabbildung den Weg in die Zukunft weist. Im Rahmen der Forschungsarbeit des Lehrstuhl fml an der TU München wurde dazu ein Konzept und Anforderungskatalog zur Ausgestaltung von Stapler-Middleware Softwaresystemen geschaffen. Eine prototypische Umsetzung zeigt die Vorteile für die Systemlieferanten, Kunden und letztendlich auch Staplerfahrer.

In der Zukunft werden flexibel agierende dezentrale Systeme weiter an Bedeutung gewinnen. Z.B. können Stapler-Leit-Systeme auch als Schwarm sich gegenseitig über aktuelle Aufträge abstimmender Flurförderzeuge realisiert werden [Göh-10]. Die vollständige Integration von Identifikationssystemen und Fahrzeugsteuerung in den Prozessfluss der Lagerverwaltungs- bzw. Stapler-Leit-Systeme erlauben es signifikante Vorteile in Prozesssicherheit und Geschwindigkeit zu erlangen. Dabei unterstützt der offen gestaltete Middleware-Konzept-Vorschlag eine Umsetzung in Wissenschaft und Praxis. Entwickler können weltweit Applikationen für den Einsatz im Lager entwickeln. Das Konzept der Stapler-Middleware kann mit einem Erfolgsbeispiel der Apple Inc. aus dem Unterhaltungselektronikbereich verglichen werden: Der Download einer sogenannten "App" aus dem AppleStore für das iPhone [App-10] hat vorgemacht, welche Fülle an Entwicklungen und Neuerungen denkbar sind, wenn man eine weltweite Entwicklergemeinschaft vernetzt und ebenso weltweit verteilten Kundenkreisen zugänglich macht. Auf gleicher Art und Weise kann eine offene Stapler-Middleware gerade kleineren Systemhäusern helfen zum Innovationstreiber zu werden.

\section{Literatur}

[App-10] Apple Inc.:

Mit jeder neuen App wird dein iPhone besser.; http://www.apple.com/de/iphone/apps-for-iphone/; Aufruf am 25.01.2010

[Bro-86] Brockhaus, F. A.:

Brockhaus Enzyklopädie, Mannheim 1986

[Erl-06] Erl, T.:

Service-oriented architecture; Prentice-Hall, Upper Saddle River, NJ 2006

[Göh-10] Göhring, S.; Lorenz, T.:

Agentenbasierte Staplerleitsysteme; In: Internet der Dinge in der

Intralogistik, Berlin. Springer, 2010

[Gün-09] Günthner, W.; Beckhaus, K.; Fischer, R.; Heinecker, M.:

(C) 2010 Logistics Journal : Nicht referierte Veröffentlichungen - ISSN 1860-5923 
Die Gabelzinke als RFID-Antenne - Auf dem Weg zu einem neuen

Standard; In: 18. Deutscher Materialfluss-Kongress; VDI-Berichte 2066, S. 43-54, Düsseldorf. VDI Verlag, 2009

[Jun-09] Jungk, A.; Heiserich, G.; Overmeyer, L.:

Antenna Concepts for RFID on Forklift Trucks; In: The 2nd International Multi-Conference on Engineering and Technological Innovation, 2009

[Las-09] Laskey, K. B.; Laskey, K.:

Service oriented architecture; In: Wiley Interdisciplinary Reviews:

Computational Statistics 2009 Heft: 1, S. 101-105

[RFi-09] RF-iT Solutions GmbH:

Produkthomepage You-R® OPEN; http://www.rf-it-

solutions.com/produkte/you-r-open.html; Aufruf am 25.01.2010

[Rie-09] Riedmaier, S.:

Navigation und Steuerung von Schmalgangstaplern mittels RFID-

Technologie; In: 15. Flurförderzeugtagung 2009 mit Fachausstellung VDIBerichte Nr. 2070, 2009

[Rie-10] Riedmaier, S.:

Ziel erreicht! - Ein Navigationsgerät für Schmalgangstapler steigert

die Effizienz um zirka 25 Prozent; In: Logistra Intralogistik 2010 Heft: 1-2

[Sil-09] Silverstroke GmbH:

Produkthomepage Tagpilot;

http://www.silverstroke.com/de/produkte/tagpilot/; Aufruf am 25.01.2010 